\title{
Miller Fischer and posterior reversible encephalopathy syndromes post COVID-19 infection
}

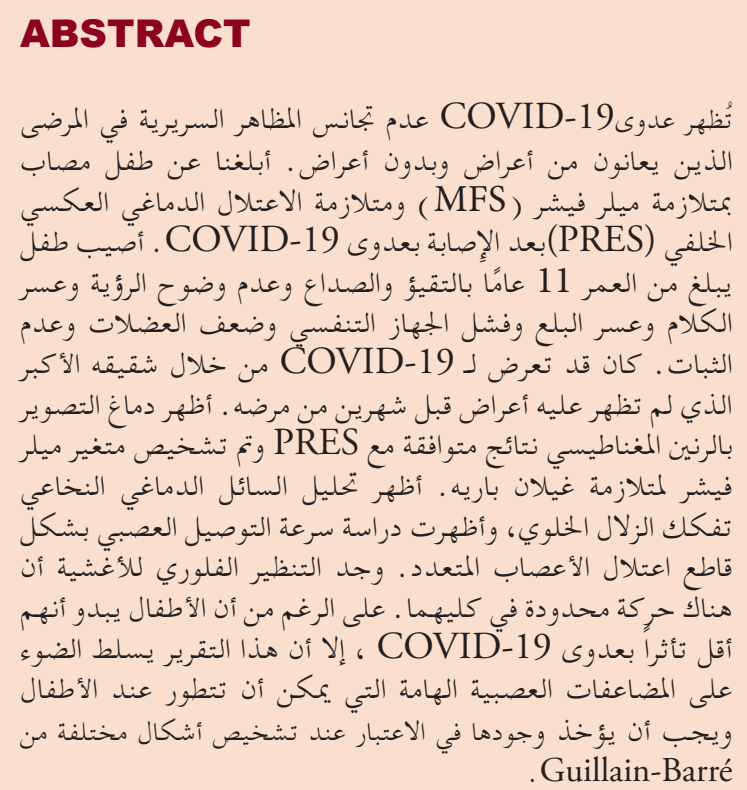

COVID-19 infection displays heterogeneity of clinical manifestations in symptomatic and asymptomatic patients. We report on a child with Miller Fischer syndrome (MFS) and posterior reversible encephalopathy syndrome (PRES) postCOVID-19 infection. An 11-year-old boy presented with vomiting, headache, blurred vision, dysarthria, dysphagia, respiratory failure, muscle weakness, and unsteadiness. He had been exposed to COVID-19 through an asymptomatic elder brother two months prior to his illness. The MRI brain showed findings consistent with PRES and the diagnosis with Miller Fischer variant of the Guillain-Barré syndrome was made. A cerebrospinal fluid analysis revealed cytoalbuminous dissociation, and a nerve conduction velocity study conclusively showed polyneuropathy. A fluoroscopy of the diaphragms found that there was limited movement in both. Although children seem to be less affected by COVID-19 infection, this report highlights on an important neurological complications that can develop in children and its presence should be taken into consideration when diagnosing different forms of Guillain-Barré.

\section{Neurosciences 2021; Vol. 26 (3): 295-299 doi: 10.17712/nsj.2021.3.20210002}

From the Department of Pediatrics, Collage of Medicine and King Khalid University Hospital, King Saud University, Riyadh, Kingdom of Saudi Arabi

Received 12th January 2021. Accepted 17th May 2021.

Address correspondence and reprint request to: Dr. Ali A. Al Haboob, Department of Pediatrics, Collage of Medicine and King Khalid University Hospital, King Saud University, Riyadh, Kingdom of Saudi Arabia.E-mail:drhbooob@gmail.com

ORCID: https://orcid.org/0000-0003-2126-7874

$\mathrm{T}$ The coronavirus disease 2019 (COVID-19) global pandemic began by the end of December 2019 in Wuhan, the capital of Hubei province in China. Millions of people have been infected worldwide. The novel coronavirus is mainly labeled as severe acute respiratory syndrome coronavirus 2 (SARS-CoV-2), it is evident that it could aggravate and or heighten other endocrine disorders such as Diabetic ketoacidosis and thyroiditis. ${ }^{1,2}$ COVID-19 is a new beta coronavirus with a hostile and injurious entry to the cell by commingling with the angiotensin-converting enzyme 2 (ACE2) receptor. $^{3}$

COVID-19 infection has been documented to result in multiple systemic affections and end organs failures, encompassed respiratory complications are the most common with externalizations simulating severe acute respiratory syndrome coronavirus (SARS-CoV). ${ }^{4}$ Other studies have reported acute renal failure, cardiovascular failure, gastrointestinal complications and hematological failure as a result ofCOVID-19 infection. ${ }^{5,6}$ Neurological manifestations have been reported, ranging from

Disclosure. The authors declare no conflicting interests, support or funding from any drug company. 


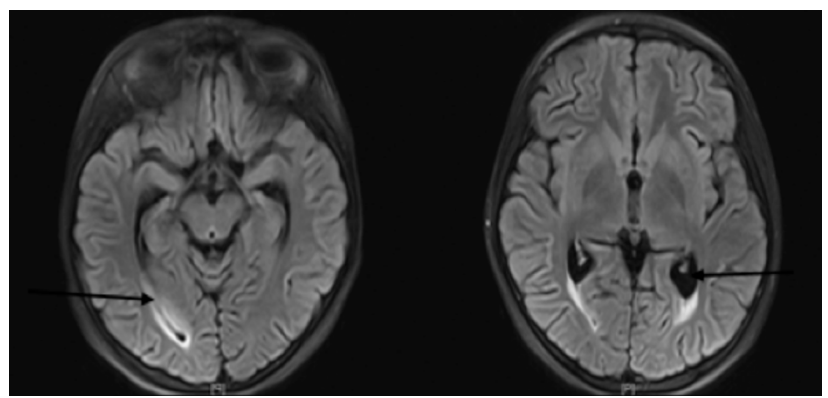

Figure 1 - Nonspecific high signal intensity surrounding the occipital horns on FLAIR images. The MRV shows a hypoplastic left lateral sinus, other venous sinuses, and deep venous system are patent. No sinus thrombosis. The MRA study is within normal limits.
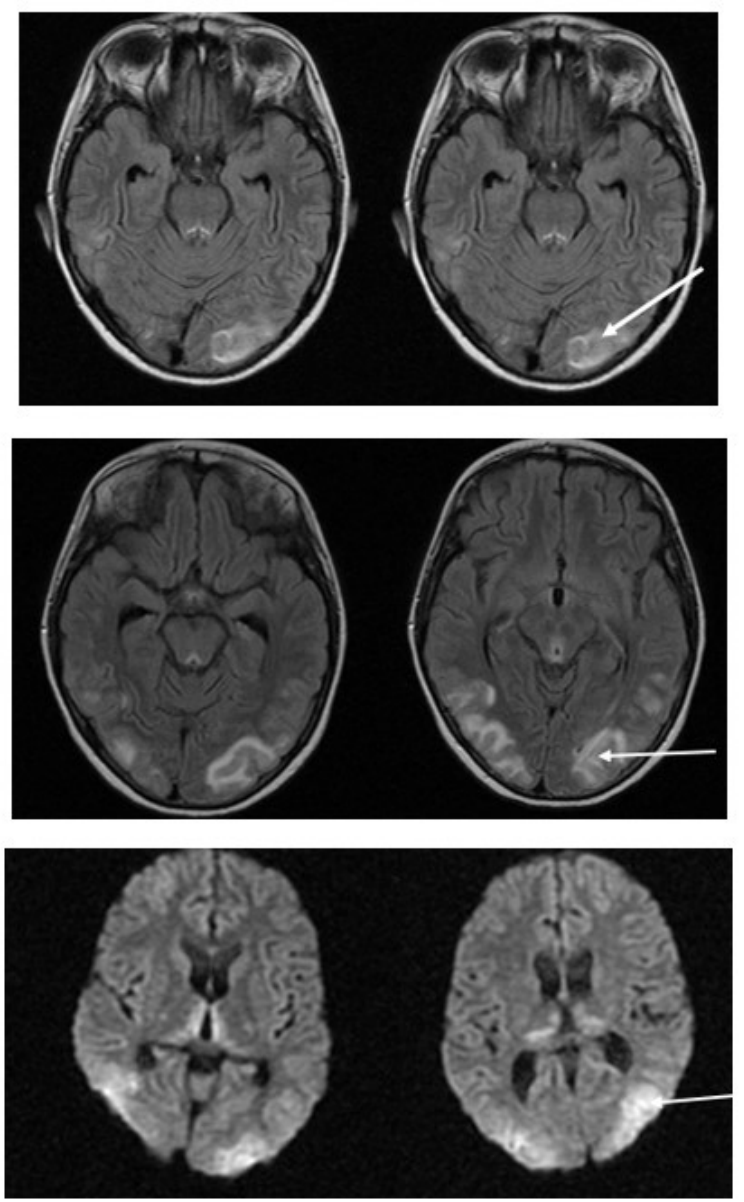

Figure 2 - Bilateral cortical and subcortical patchy high signal intensity on FLAIR and T2W sequences affecting parieto occipital areas with mild involvement of frontal areas (at watershed zones) in both thalami. No diffusion restriction. Unremarkable MRA and MRV.

headache, dizziness, hyposmia, hypogeusia, muscle damage and weakness to cerebrovascular strokes. ${ }^{7}$ Additional neurological complications includes viral

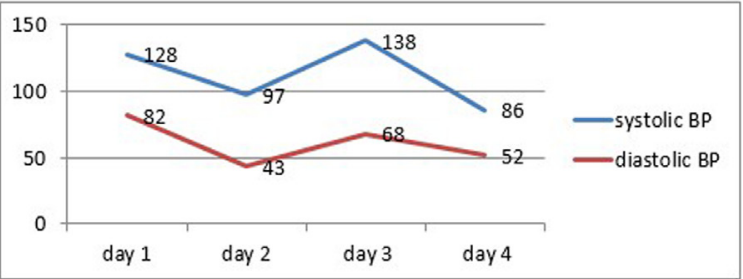

Figure 3 - Systolic and diastolic blood pressure (BP) recorded during the first 4 days admission, showing fluctuation in both the systolic and diastolic BP.
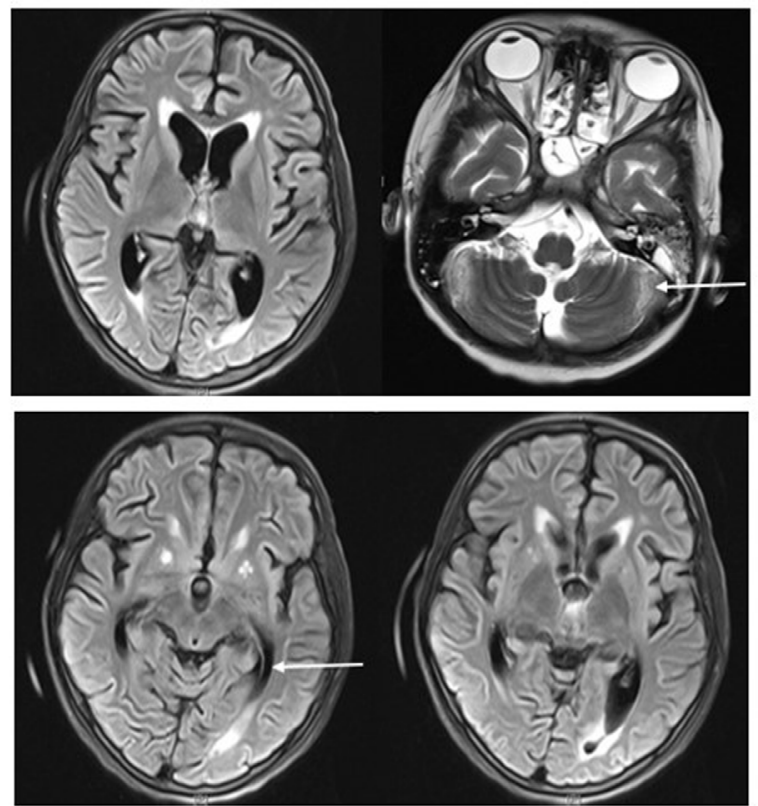

Figure 4 - Interval resolution of the previously noted lesions of PRES. Progressive moderate global cerbral and cerebellar atrophic changes and periventricular white matter gliosis. Bilateral putaminal T1 hyperintensities. Unremarkable MRA brain scan.

encephalitis, acute necrotizing encephalitis, and acute disseminated encephalitis have been reported. ${ }^{5,8,9}$

In pediatric age groups, COVID-19 infection has been reported to be mild for the enormous majority of affected children with severe infection in other cases and with differences in the clinical embodiment as resembled to adult patients. There was an initial report of a presentation similar to incomplete Kawasaki disease (KD) or toxic shock syndrome in the United Kingdom, ${ }^{10}$ which was later followed by reports of similarly affected children in different parts of the world. Different names have been used for this disarray, such as multisystem inflammatory syndrome in children (MIS-C), pediatric 
An 11-year-old male child who was previously healthy with an unremarkable medical history

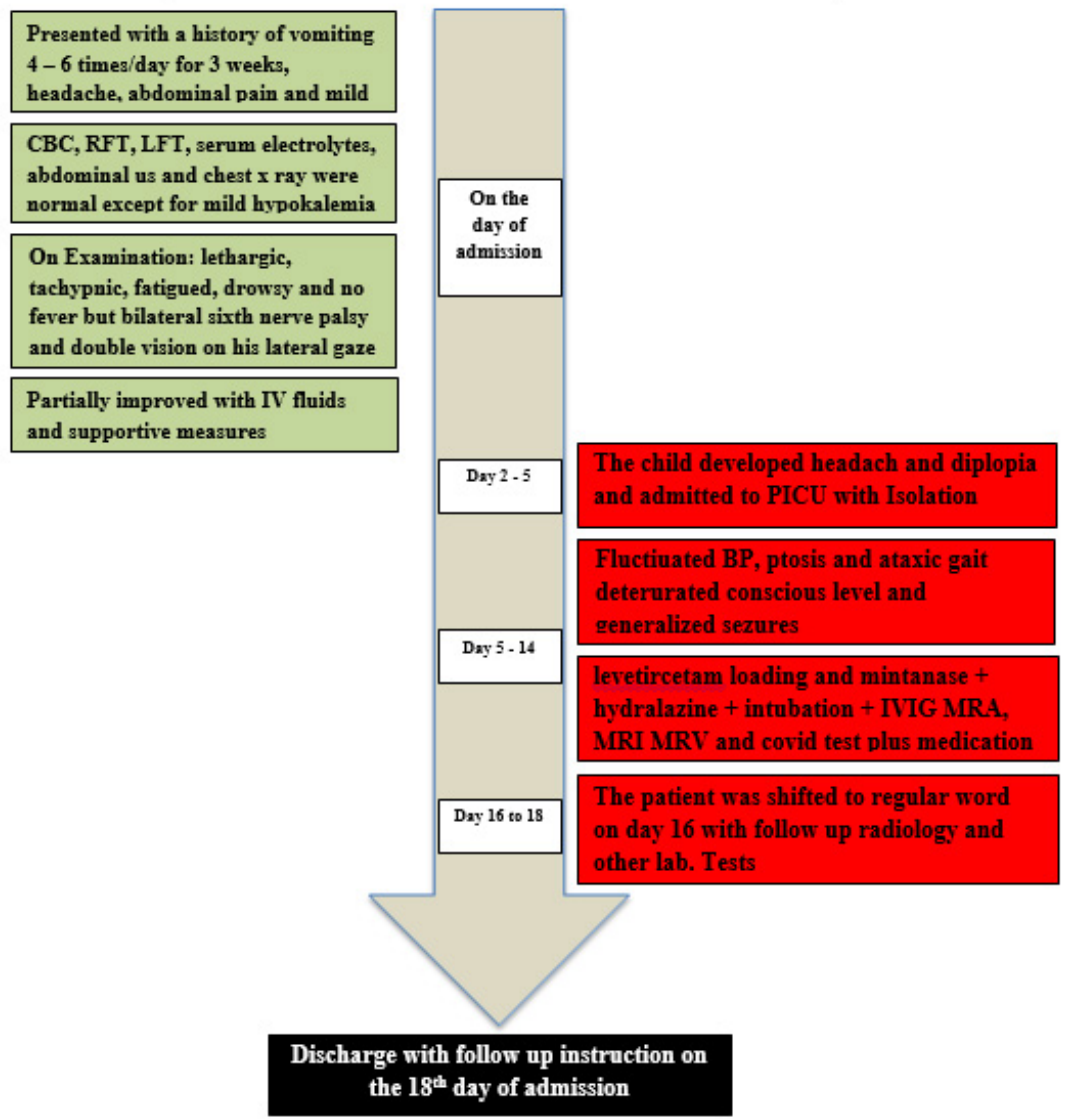

Timeline flowchart, describing the patient journey since presentation to ER and throughout his hospital course until discharge

Figure 5 - Timeline flowchart.

multisystem inflammatory syndrome (PMIS), pediatric multisystem inflammatory syndrome temporally associated with SARS-CoV-2 (PIMS-TS), pediatric hyper inflammatory syndrome, or pediatric hyper inflammatory shock. ${ }^{10}$

Miller Fischer syndrome, which is a rare variant of GBS that is characterized by bulbar palsy and phrenic nerve palsy and a clinical triad of ophthalmoplegia, ataxia and areflexia, has not yet been reported as a COVID-19-associated condition in children. Hence, the report considered in this paper, which is for a child with Miller Fischer Syndrome associated with posterior reversible encephalopathy syndrome (PRES) secondary to COVID-19 infection, presents a rare instance of such an association.
Case Report. Patient information. The current report is for an 11-year-old male child who was previously healthy with an unremarkable medical history. He presented at our hospital with a history of vomiting that had started 3 weeks prior to presentation. He was vomiting approximately 4 to 6 times a day, and it was effortless and non-projectile, associated with abdominal pain and mild diarrhea for a few days without any constipation, and he had mild headache. There was no history of fever. The child was first evaluated at a primary health care facility where he had a computerized tomography (CT) brain scan, a serum electrolytes test, a renal profile, liver functions tests, a complete blood count test, an abdominal ultrasonography, and a chest x-ray. All were unremarkable except for mild hypokalemia. The child was given intravenous fluid 
therapy and non-specific supportive measures. There was no documentation for any COVID-19 workup at that time. The child's condition partially responded to treatment for a few days, but shortly after, the vomiting recurred with mild headache, and he started to complain of diplopia.

Clinical findings. The initial evaluation at our hospital revealed that the boy had normal vital signs for his age, was afebrile, and was fully conscious with a Glasgow Coma Scale (GCS) score of 15/15; he had normal orientation to persons, time, and place and a normal fundus examination, although he displayed bilateral sixth nerve palsy and double vision on his lateral gaze. He had normal muscle tone and power, normal deep tendon reflexes, and the rest of his physical examination was unremarkable.

Diagnostic assessment. The initial workup included a complete blood count, a renal profile, liver function tests, an erythrocyte sedimentation rate (ESR) test, a c-reactive protein (CRP) test, and his levels of serum electrolytes and procalcitonin were measured. Magnetic resonance imaging (MRI), magnetic resonance angiography (MRA), and magnetic resonance venography (MRV) of the brain were also completed (Figure 1). The child was admitted to the pediatric intensive care unit (PICU) for close monitoring, follow-up, and management, and the hospital policy for isolation precautions was followed.

Therapeutic intervention. The child was started on intravenous immunoglobulins (IVIG) at a dose of 0.4 $\mathrm{gm} / \mathrm{kg} /$ day for 5 days to treat the rare MFS variant of GBS. By the second day of hospitalization, the child's blood pressure began fluctuating between hypertensive and hypotensive states (Figure 3). $\mathrm{He}$ remained afebrile, was still fully conscious with a GCS score of $15 / 15$, and had evident bilateral ptosis and ataxic gait. Over the course of the day, his level of consciousness deteriorated to GCS 8/15 and he developed generalized tonic-clonic seizures, which were controlled with levetircetam loading, and he was kept on maintenance doses. His neurological examination revealed that his pupils were $2-3 \mathrm{~mm}$ in length, both were equal, and they were reactive to light. He had ophthalmoplegia, weak gag reflex, absent cough, and absent deep tendon reflexes. The child was orally intubated and connected to mechanical ventilation. His temporary hypertension episodes were managed with hydralazine $(0.1 \mathrm{mg} / \mathrm{kg} \mathrm{Q}$ 4 hours PRN).

Follow-up and outcomes. Testing for the new SARS-COVID-19 real-time polymerase chain reaction (RT-PCR) was completed, and the result was positive. Furthermore, a workup for the possibility of COVID-19 associated multisystem inflammatory syndrome in children (MIS-C) was completed, which included checking ESR, CRP, procalcitonin, serum ferritin, triglycerides, blood lactate, lactic dehydrogenase, d-dimer, cardiac troponin-t, $\mathrm{N}$-terminal pro b-type natriuretic peptide (NTproBNP), and interleukin-6 (IL-6).

The diagnosis of Miller Fischer syndrome (MFS) with posterior reversible encephalopathy syndrome in association with COVID-19 infection was made based on clinical presentation, radiological features, and the findings of a nerve conduction study.

Levetiracetam was administered at a maintenance dose for control of convulsions, hydralazine was used to control blood pressure, and the child was kept on mechanical ventilation and provided with other required supportive measures according to his progress. The hospital guidelines for management of COVID-19 in pediatric age groups at that time were also followed, and this involved administering broad spectrum antibiotics (ceftriaxone and azithromycin), remdesivir (with a loading dose of $5 \mathrm{mg} / \mathrm{kg}$ and maintenance dose of $2.5 \mathrm{mg} / \mathrm{kg} /$ day for 10 days), methylprednisolone (30 $\mathrm{mg} / \mathrm{kg}$ once daily for 3 days), and a small dose of acetyl salicylic acid ( $5 \mathrm{mg} /$ day).

A lumbar puncture for cerebrospinal fluid (CSF) was carried out and showed no cells and a protein count of $0.8 \mathrm{mg} / \mathrm{ml}$ (the normal range is $0.15-0.45 \mathrm{mg} / \mathrm{ml}$ ) confirming the CSF cytoalbuminous dissociation. A gram stain showed no organisms, and a later CSF culture was negative. A nerve conduction velocity study was also completed, and the results were abnormal. A chest $\mathrm{x}$-ray, a CT scan of the chest, a 12-lead electrocardiogram (ECG), and an echocardiography were also carried out, and all were reported to be normal. Furthermore, an urgent MRI, MRA, and MRV of the brain were repeated and confirmed a deteriorating condition (Figure 2).

Over the following 14 days, the child's vital signs stabilized with no further blood pressure fluctuation, he remained afebrile, the convulsions were under control, the ophthalmoplegia improved, and gag and cough reflexes were regained gradually. The child's muscle tone showed significant improvement, and deep tendon reflexes were elicited. A follow-up RT-PCR for COVID-19 was carried out on 2 occasions, on day 5 and day 7 since the initiation of treatment, and both were negative. Follow-up MRI, MRA, and MRV brain scans indicated that the previously reported lesions had resolved. Further follow-up on inflammatory markers showed significant improvement of the initially elevated markers. 
The child's gas exchange was maintained on minimal ventilation settings, and he was successfully intubated, initially to non-invasive positive pressure support and later to simple nasal cannula oxygen. Finally, the child was discharged from the PICU to the general ward and later to home with a normal level of consciousness and orientation, no apparent cranial nerve palsy, normal muscle tone, grade 4 motor power, and normal gag and cough reflexes.

Discussion. The PRES is associated with immunosuppressive and cytotoxic therapies. Prior exposure to a predisposing drug does not appear to be protective, and patients can develop PRES even several weeks after exposure. ${ }^{2}$ In addition, the disorder has been associated with both acute and chronic renal disease, as was the case for our patient, and medical conditions, such as hyponatremia or pulmonary infection, could exacerbate the neurological findings (Figure 4).

Although it has not been reported yet, the occurrence of PRES a few days after the surge of interleukin (IL-6 or IL-1) associated with COVID infection pathophysiology suggests that these kinds of immunomodulatory agents may also lead to PRES.

Guillain-Barré syndrome (GBS) and its rare variants, such as Miller Fischer Syndrome, have also been reported to occur secondary to COVID-19 infection in case series from different parts of the world; ${ }^{3,9}$ however, these case series were all for adult patients with few reports of COVID-19 associated GBS in children. ${ }^{4}$

In conclusion, cautious management of patients presenting with neurological manifestations with a COVID +ve results is highly advisable including complete neurological examination, CSF analysis, radiology and aggressive therapeutic intervention. Moreover, future researches on the pathology of COVID -19 and neurological manifestations coexistence is highly advisable.
Acknowledgment. We would like to thank Scribendi (https://www.scribendi.com/) for English language editing and Dr. Mazen Abdulrahman Binmujlli for reviewing the radiological images.

\section{References}

1. Zito A, Alfonsi E, Franciotta D, Todisco M, Gastaldi M, Cotta Ramusino MC, et al. COVID-19 and Guillain-Barré Syndrome: A Case Report and Review of Literature. Front Neurol 2020; 11: 909.

2. Zhao Y, Zhao Z, Wang Y, Zhou Y, Ma Y, Zuo W. Single-cell RNA expression profiling of ACE2, the putative receptor of Wuhan 2019-nCov. Am J Respir Crit Care Med 2020; 202: 756-759.

3. Huang C, Wang Y, Li X, Ren L, Zhao J, Hu Y, et al. Clinical features of patients infected with 2019 novel coronavirus in Wuhan, China. Lancet 2020; 395: 497-506.

4. Chen L, Liu HG, Liu W, Liu J, Liu K, Shang J, et al. [Analysis of clinical features of 29 patients with 2019 novel coronavirus pneumonia]. Zhonghua Jie He He Hu Xi Za Zhi 2020; 43: E005.

5. Mao L, Jin H, Wang M, Hu Y, Chen S, He Q, et al. Neurologic Manifestations of Hospitalized Patients With Coronavirus Disease 2019 in Wuhan, China. JAMA Neurol 2020; 77: 683-690.

6. Sahin AR, Erdogan A, Mutlu Agaoglu P, Dineri Y, Cakirci AY, Senel ME, et al. 2019 Novel Coronavirus (COVID-19) Outbreak: A Review of the Current Literature. EJMO 2020; 4 : 1-7.14.

7. Riphagen S, Gomez X, Gonzalez-Martinez C, et al. Hyperinflammatory shock in children during COVID-19 pandemic. Lancet 2020; 395: 1607-1608.

8. Feldstein LR, Rose EB, Horwitz SM, et al. Multisystem Inflammatory Syndrome in U.S. Children and Adolescents. $N$ Engl J Med 2020; 383: 334-346.

9. Marino S, Marino L, Greco F, Venti V, Fontana A, Timpanaro $\mathrm{T}$, et al. Bickerstaff's brainstem encephalitis in childhood: a literature overview. Eur Rev Med Pharmacol Sci 2020; 24 : 12802-12807.

10. L. Diez-Porras, E. Vergés and F. Gil et al., Guillain-BarréStrohl syndrome and COVID-19: Case report and literature review, Neuromuscular Disorders, https://doi.org/10.1016/j. nmd.2020.08.354. 